\title{
Our panel of experts highlight the most important research articles across the spectrum of topics relevant to the field of hematologic oncology
}

Expert panel: Stefano Molica, General Hospital "Pugliese-Ciaccio", Catanzaro, Italy; Pamela S Becker, University of Washington, WA, USA

\begin{abstract}
Mesa RA, Gotlib J, Gupta V et al. Effect of ruxolitinib therapy on myelofibrosis-related symptoms and other patient-reported outcomes in COMFORT-I: a randomized, doubleblind, placebo-controlled trial. J. Clin. Oncol. doi:10.1200/JCO.2012.44.4489 (2013) (Epub ahead of print).
\end{abstract}

There are many types of patient-reported outcome tools used in oncology. The myelofibrosis symptom assessment form (MFSAF) was developed to evaluate the presence and severity of myelofibrosis-related symptoms. A more simplified version of the MFSAF was later developed and used in a Phase III, double-blind, placebo-controlled study (COMFORT-I). In the present analysis Mesa et al. assessed the ability of the modified MFSAF to measure significant changes in myelofibrosis-related symptoms and the effects of ruxolitinib on symptom burden.

Patients who received ruxolitinib experienced improvements in individual myelofibrosis-related symptoms. The majority (91\%) of ruxolitinib-treated patients experienced a $\geq 50 \%$ improvement of total symptom score (TSS). Patients with $\geq 50 \%$ TSS response considered their condition as either 'much improved' or 'very much improved'. These patients achieved significant improvements in the European Organisation for Research and Treatment of Cancer Quality of Life Questionnaire Core 30 (EORTC QLQ-C30) functional domains and global health status/quality of life in comparison with patients receiving placebo, who experienced worsening on these measures $(\mathrm{p}=0.0135)$. The degree of spleen volume reduction with ruxolitinib correlated with improvements in TSS, fatigue scale score and EORTC global health status/quality of life score.

In conclusion, changes in the modified MFSAF score with ruxolitinib therapy were clinically relevant. In addition, the modified MFSAF score correlated well with established patient-reported outcome measures. Even patients with modest changes in spleen size or symptom scores demonstrated improvements in symptom burden and quality of life, whereas patients receiving placebo continued to worsen by these same measures. These results support the use of the MFSAF in clinical studies of treatments for myelofibrosis.

- Written by S Molica

Rossi D, Rasi S, Spina V et al. Integrated mutational and cytogenetic analysis identifies new prognostic subgroups in chronic lymphocytic leukemia. Blood 121(8), 1403-1412 (2012).

New somatic mutations (i.e., NOTCH1, SF3B1 and BIRC-3) have been described in chronic lymphocytic leukemia (CLL), however, a relevant issue is how they can be integrated into the prognostic stratification based on cytogenetic analysis.

In this study Rossi et al. proposed a comprehensive and dynamic prognostic algorithm including gene mutations, chromosomal abnormalities and their changes during clonal evolution. For this purpose

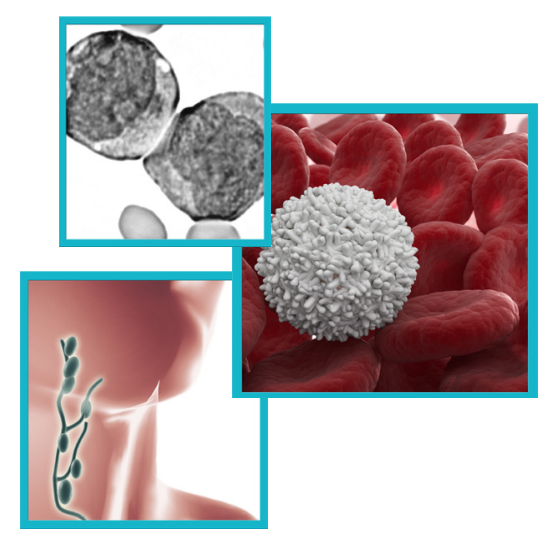

News \& Views

News

Journal Watch

Ask the Experts

Interview 
1274 CLL samples were analyzed. Using both a training validation and a timedependent design, the authors identified four CLL patient subgroups who could be classified:

- High risk, harboring TP53 and/or BIRC3 abnormalities;

- Intermediate risk, harboring NOTCH1 and/or SF3B1 mutations and/or del11q22-q23;

- Low risk, harboring trisomy 12 or normal genetics;

- Very low risk, harboring del13q14 only.

This classification provided a hierarchic survival pattern; in fact, 10 -year survival rates were as follows: high risk, 29\%; intermediate risk, 37\%; low risk, 57\%; and very low risk, 69.3\%. Interestingly, for verylow-risk patients, overall survival did not significantly differ from a matched general population.

This integrated mutational and cytogenetic model, which independently predicted survival, was also validated in an independent CLL cohort. Furthermore, by taking into account clonal evolution over time, this genetic model maintained its prognostic relevance at any time from diagnosis.

These data show that the integrated mutational and cytogenetic model can help to classify CLL patients into more specific subgroups, thus improving current prognostic algorithms. Future challenges are the validation of these concepts in prospective trials aimed at investigating whether specific treatments may overcome the poor prognosis conferred by higher-risk lesions.

- Written by S Molica

\section{Dimopoulos MA, Roussou M, Gkotzamanidou $\mathrm{M}$ et al. The role of novel agents on the reversibility of renal impairment in newly diagnosed symptomatic patients with multiple myeloma. Leukemia 27(2), 423-429 (2012).}

Renal impairment (RI) is present in approximately $50 \%$ of multiple myeloma
(MM) patients at some point in the course of their disease. As it is the most common cause of early mortality in MM, patients with RI during initial therapy should be considered high risk, especially during induction, with myeloma cast nephropathy the most common cause of serious renal compromise. The role of thalidomide, bortezomib and lenalidomide in MM patients presenting with RI was evaluated by Dimopoulos et al. in 133 newly diagnosed patients who were treated with a novel agent-based regimen. In RI patients bortezomib worked better than thalidomide or lenalidomide. In fact, a significant improvement of renal function (renal partial response) was observed in $77 \%$ of patients treated with bortezomib, in $55 \%$ with thalidomide and in $43 \%$ with lenalidomide ( $\mathrm{p}=0.011$ ). Renal partial response was obtained in patients treated with bortezomib in a median time of 1.34 versus 2.7 months for thalidomide and $>6$ months for lenalidomide-treated patients $(p=0.028)$. In multivariate analysis bortezomib-based therapy, higher doses of dexamethasone (160 mg during the first month of treatment), an estimated glomerular filtration rate of $30 \mathrm{ml} / \mathrm{min}$ and an age of 65 years were independently associated with shorter time to renal response. In conclusion, bortezomib-based therapies may be more appropriate for the initial management of patients with myeloma-related renal failure, however, thalidomide and lenalidomide are also associated with significant probability of improvement of their renal function.

Although RI has traditionally been known to be an adverse prognostic and survival factor, new treatments and novel combinations may be changing this as the treatment paradigm in MM continues to evolve. Use of these newer agents in the first-line therapy of MM patients with RI is recommended, provided dose reduction is used when indicated. Future large-scale studies are needed to compare the efficacy and safety of these newer drugs in different novel combinations for use in patients with MM and RI.

- Written by S Molica
Marks DI, Moorman AV, Chilton Let al. The clinical characteristics, therapy and outcome of 85 adults with acute lymphoblastic leukemia and $t(4 ; 11)$ (q21;q23)/MLL-AFF1 prospectively treated on UKALLXII/ECOG2993. Haematologica doi:10.3324/ haematol.2012.081877 (2013) (Epub ahead of print).

The prognosis of adults with acute lymphoblastic leukemia (ALL) has been noted to be worse than pediatric patients, with a worse long-term outcome with chemotherapy regimens. The decision as to which adult patients should be considered for allogeneic hematopoietic cell transplant in first complete remission has changed in the last few years, and it has been critical to determine which prognostic groups have a worse outcome. It has long been appreciated that the patients who have the $B C R-A B L$ translocation do not remain in remission after conventional induction, consolidation and maintenance treatment, but the prognosis of other translocations is only just beginning to be recognized.

MLL1 is the mixed lineage leukemia gene at 11q23, and it has been found to be involved in poor prognosis leukemias, particularly in infant acute lymphoblastic leukemia. The $\mathrm{t}(4 ; 11)$ translocation is the second most frequent translocation in adult ALL, compromising approximately $8-10 \%$ of precursor B-cell ALL cases. The prognosis of a large number of patients with $t(4 ; 11)$ adult ALL was determined after treatment on protocol UKALLXII/ECOG2993. Of the $913 B C R-A B L$-negative patients with precursor B-cell ALL enrolled on the study, 85 had the $\mathrm{t}(4 ; 11)$ translocation. The complete remission rate was $93 \%$ overall; it was better for patients with a lower initial white count $(100 \%)$ than for those with a higher initial white count $(89 \%$, the white blood cell count limit was set at $\left.100 \times 10^{\circ} / \mathrm{l}\right)$. At a median follow-up of 5.4 years, the 5 -year event-free survival was $34 \%$. The 5 -year overall survival was superior in those who received a syngeneic or allogeneic transplant (56\%), compared with those who had chemotherapy or an autologous transplant (24\%). For patients aged $40-59$ years, the overall survival and event-free survival were 
particularly poor, as each was only $19 \%$. The outcomes for patients who had relapsed ALL was also extremely poor. A multivariate analysis showed that age was correlated with outcome, regardless of postremission treatment. The phenotype of the $t(4 ; 11)$ negative and -positive leukemia cells differed significantly with respect to the following antigens: CD34, CD10, CD133, CD135, CD105, CD65 and CD15. The authors suggest that the $\mathrm{t}(4 ; 11)$-positive leukemias may have a less-differentiated status, and they expressed the last two antigens, which were myeloid, and had high expression of Flt3. They found that there was a lack of additional genomic aberrations, and describe that $M L L 1$-rearranged cases exhibit epigenetic dysregulation. They propose that the ongoing CALGB 10403 trial, being conducted as an intergroup study, will eventually provide data on the outcomes of $t(4 ; 11)$ adolescent and young adult patients treated on a pediatric-type protocol. They also describe the approach of the UKALL2011 trial in the UK, which will allow patients aged $15-24$ years with $t(4 ; 11)$ and with minimal residual disease present at 14 weeks to proceed to allogeneic hematopoietic cell transplant in first remission. Thus, this publication affirms the relatively worse prognosis of the $t(4 ; 11)$ translocation, especially in the patients aged 40 years and older, or in the relapsed setting, and these prognostic data should be considered when determining treatment algorithms incorporating decisions regarding candidacy for allogeneic hematopoietic cell transplant for adult ALL.

- Written by PS Becker

Gaymes TJ, Mohamedali AM,
Patterson M et al. Microsatellite
instability induced mutations in
DNA repair genes CtIP and MRE11
confer hypersensitivity to poly
(ADP-ribose) polymerase (PARP)
inhibitors in myeloid malignancies.
Haematologica doi:10.3324/
haematol.2012.079251 (2013) (Epub
ahead of print).

DNA repair is fundamental to maintenance of the accuracy of the genetic code. There are a number of DNA repair pathways involved, including the DNA mismatch repair pathway. Members of this pathway maintain the fidelity of repeat sequences, such as those in microsatellite DNA. Mutations in microsatellite DNA lead to microsatellite instability (MSI), which contributes to the so-called mutator phenotype, characterized by accumulation of mutations that leads to cancer progression. Chromosome instability is a hallmark of myelodysplastic syndrome (MDS) and acute myeloid leukemia (AML), and it is well known that there are chromosome deletions, translocations and additions in these disorders. Certain chemotherapy drugs cause dsDNA breaks, and even overactivity of the nonhomologous end-joining DNA damage response pathway can lead to joining errors. Such defects render the cells susceptible to treatment with PARP inhibitors.

This study demonstrates the presence of MSI with monoallelic mutations in CtIP and MRE11 in samples from myelodysplastic syndrome MDS/AML cell lines and patients with AML. First, mutations were identified within six mononucleotide repeat markers using fluorescent fragment PCR. Eleven percent of the primary AML samples exhibited MSI by this method. DNA sequencing of the coding-region mononucleotide repeats within the DNA repair genes $B L M, A T R$, DNA-PK, ATM, MRE11, CtIP, RAD50, CHK1, BRCA1, BRCA2, PARP1, TNK1 and PTEN and in the gene, TGF- $\beta I I$ revealed mutations in MRE11 and CtIP, each in one patient sample and in several cell lines. Western blotting confirmed the presence of a truncated form of MRE11 and a twofold reduction in the CtIP level in the patient samples with the respective mutations. In addition, 13 out of 63 MDS patient samples exhibited MSI and also had chromosomal instability, while $15 \mathrm{MDS}$ patients without MSI exhibited no chromosomal abnormalities. Four out of 11 MDS patient samples with MSI had monoallelic mutations in CtIP. Samples from patients and cell lines that exhibited MSI also showed downregulation of homologous recombination DNA repair and conferred sensitivity to PARP inhibitors. Some AML patient samples and cell lines exhibited in vitro sensitivity to a PARP inhibitor, BMN 673.
Identification of the role of DNA repair in progression of MDS/AML may lead to new targeted therapy and, in fact, there is already an ongoing Phase I clinical trial at Kings College London (London, UK; ClinicalTrials Identifier: NCT01399840) that is examining the efficacy of PARP inhibitors in the treatment of MDS/AML.

- Written by PS Becker

\section{Shih AH, Chung SS, Dolezal EK et al. Mutational analysis of therapy- related myelodysplastic syndromes and acute myelogenous leukemia. Haematologica doi:10.3324/ haematol.2012.076729 (2013) (Epub ahead of print).}

Therapy-related myelodysplastic syndrome (MDS) and acute myeloid leukemia (AML) develop in patients after treatment with chemotherapy with or without radiation, including patients who have undergone autologous stem cell transplant. These secondary complications are invariably associated with poor prognosis and drug resistance. This study examined samples from patients with these disorders for the presence of somatic mutations in key genes associated with MDS/AML. The blast fractions were isolated by FACS or immunomagnetic beads. Exon sequencing was performed for TP53, TET2, DNMT3A, ASXL1, IDH1, IDH2, EZH2, EED, SUZ12, RBBP4, SRSF2, U2AF35 and SF3B1. The most commonly observed mutations were in TP53 and TET2, occurring in 21\% (eight out of 38 ) and $10 \%$ (four out of 38) of cases, respectively, with mutations in the other genes occurring with a frequency of $<10 \%$. Of the cases with a mutation or loss of TP53, there was a high incidence of complex cytogenetics $(91 \%)$ or concomitant deletion of chromosome 5 or $5 \mathrm{q}(83 \%)$. Moreover, the median survival of patients with mutated or deleted TP53 was only 8.8 months, compared with 37.4 months $(\mathrm{p}=0.0035)$ for those with wild-type TP53. The authors note that the frequency of abnormalities in TP53 is much higher than they have observed in MDS/AML that has not arisen after chemoradiotherapy. The molecular 
alterations reported here provide insight into the biology of secondary MDS/AML and have a potential impact on therapy for these disorders.

- Written by PS Becker
Financial \& competing interests disclosure

The authors have no relevant affiliations or financial involvement with any organization or entity with a financial interest in or financial conflict with the subject matter or materials discussed in the manuscript. This includes employment, consultancies, honoraria, stock ownership or options, expert testimony, grants or patents received or pending, or royalties.

No writing assistance was utilized in the production of this manuscript.

Journal Watch highlights some of the most important papers recently published in the field of hematologic oncology and research. The editorial team welcomes recommendations for relevant papers for inclusion in future issues.

Please direct your suggestions to:

Roshaine Gunawardana, Managing Commissioning Editor, International Journal of Hematologic Oncology

r.gunawardana@futuremedicine.com 